\title{
Watermarking of 3D Irregular Meshes based on Wavelet Multiresolution Analysis ${ }^{\star}$
}

\author{
Min-Su Kim ${ }^{1,2}$, Sébastien Valette ${ }^{3}$, Ho-Youl Jung ${ }^{2}$, and Rémy Prost ${ }^{1}$ \\ 1 CREATIS, INSA-Lyon, France, \\ \{kim, prost\}@creatis.insa-lyon.fr \\ 2 MSP Lab. Yeungnam University. Korea \\ hoyoul@yu.ac. $k r^{\star \star}$ \\ 3 Informatics \& Telematics Institute, Thessaloniki, Greece \\ valette@iti.gr
}

\begin{abstract}
In this paper, we propose a robust watermarking method for 3 -D triangle surface meshes. Most previous methods based on the wavelet analysis can process only semi-regular meshes. Our proposal can be applied to irregular as well as regular meshes by using recently introduced irregular wavelet analysis scheme. L2-Norm of the wavelet coefficients is modified in various multi-resolution levels to embed the watermark. We also introduced a vertex and face re-ordering process as pre-processing in both watermark embedding and extraction for the robustness against connectivity reordering attacks. In addition, our proposal employs blind watermark detection scheme, which can extract the watermark without reference of cover mesh model. Through the simulations, we prove that our approach is robust against connectivity reordering as well as various kinds of geometrical attacks such as lossy compression and affine transform.
\end{abstract}

\section{Introduction}

Outstanding progress of digital multimedia data has eased its reproduction and retransmission. Such trend also increases the need for copyright protection. Traditional data protection techniques such as encryption are not adequate for copyright enforcement, because the protection cannot be ensured after the data is decrypted. Unlike the encryption, digital watermarking does not restrict access to the host data, but ensures the hidden data to remain inviolated and recoverable $[1,2]$. Watermarking is a copyright protection technique to embed information, so-called watermark, into host data.

Most of previous watermarking technologies have focused on traditional media data, such as digital text, audio, image, and video data. Recently, 3-D geometric models including Computer Aided Design (CAD) based 3-D data, Virtual

\footnotetext{
* This work was supported by both Korea Science and Engineering Foundation (KOSEF, 000-B-105-898) and Centre National de la Recherche Scientifique, France (CNRS, 14894).

** Corresponding author. Tel.: +82-53-810-3545, Fax: +82-53-810-4742 (Ho-Youl Jung)
} 
Reality Modeling Language (VRML), and MPEG-4, have been receiving a lot of attention, due to powerful computational performance of today's computer and the demand for a better representation of virtual worlds and scientific data. Despite such popularity, few watermarking methods have been proposed for 3-D geometric model. This is caused in part by the watermarking technology that has emerged for image, video, and audio which cannot be easily adapted to work for 3-D geometric models [3].

Since the watermarking technique for 3D meshes was introduced in [4], there have been several trials to improve the performance in terms of capacity, invisibility and robustness. Praun et al. [3] generalized the spread-spectrum approach commonly used in 2D image watermarking [1] to 3D surface meshes. Yin et $a l$. [5] extends [3] by using the half-edge collapse method. It has lower complexity than edge collapse to build multiresolution meshes. They can embed 250 bits into coarser mesh while having robustness against several attacks including geometrical and topological attacks. However, it requires a registration before an extraction to be robust against similarity transform: rotation, uniform scaling and translation. It also needs resampling of both the original mesh and the watermarked after simplification, vertex reordering or cropping attacks. It is a non-blind watermarking because the registration and the resampling need the original mesh. It is encouraged not to use the original mesh in detection for copyright protection systems. Cho et al. [6] proposed a blind watermarking method. They modify the distribution of vertex norms which is invariant to similarity transform and vertex reordering. The method is robust against various attacks.

Kanai et al. [7], in 1998, proposed the first watermarking method based on wavelet analysis with non-blind detection scheme. Uccheddu et al. [8] extends [7] to detect the watermark without the original mesh. Both of them cannot process irregular meshes directly because of the limitation of Lounsbery's scheme [9]. They can embed the watermark into an irregular mesh by using remeshing that converts an irregular mesh into a semi-regular one. But, the remeshed model cannot be seen as identical to the original, as it corresponds to a different sampling of the underlying 3D surface : the mesh connectivity is different from the original.

In this paper, we propose a watermarking method for 3D meshes, which allows to embed the information data at various resolution levels, and to detect the watermark without the original mesh. Our multiresolution approach is more effective than spatial domain one in the viewpoint of invisibility, capacity and robustness. Our proposal is based on irregular wavelet analysis scheme, recently introduced as wavelet analysis technique for both regular and irregular meshes [10], so that our watermarking method can be applied to both regular and irregular 3D triangular meshes. In our proposal, the watermark is embedded by modifying L2-norm of the wavelet coefficients.

The rest of this paper is organized as follows. In Section 2.1, we describe briefly wavelet based multiresolution analysis for irregular 3-D surface meshes. We explain the synchronization issue caused from the wavelet approach of 3-D meshes and give our approach to overcome the problem in Section 2.2. Section 


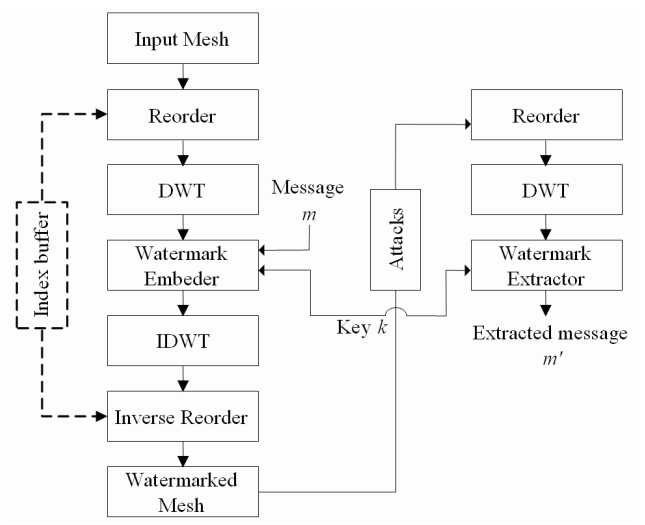

Fig. 1. Watermark embedding and extraction of our approach

2.3 and 2.4 presents the embedding and the extraction method. Simulation results show the effectiveness of our proposal in Section 3. Finally, we draw a conclusion.

\section{Our approach}

\subsection{Wavelet multiresolution analysis of irregular meshes}

Irregular wavelet analysis scheme [10] simplifies the original mesh by reversing an irregular subdivision scheme. The simplification is repeated until the resulting mesh cannot be simplified anymore. For meshes homeomorphic to a sphere, the simplest mesh is a tetrahedron. We obtain a hierarchy of meshes from the simplest one $M^{0}$, called base mesh, to the original mesh $M^{J}$. Following [10], the wavelet decomposition can be applied to the geometrical properties of the different meshes which are linked by the following matrix relations:

$$
\begin{gathered}
C^{j-1}=A^{j} C^{j} \\
D^{j-1}=B^{j} C^{j} \\
C^{j}=P^{j} C^{j-1}+Q^{j} D^{j-1}
\end{gathered}
$$

where $C^{j}$ is the $v^{j} \times 3$ matrix representing the coordinates of the vertices of $M^{j}, v^{j}$ is the number of vertices for each mesh $M^{j} . D^{j-1}$ is the $\left(v^{j}-v^{j-1}\right) \times 3$ matrix of the wavelet coefficients at level $j . A^{j}$ and $B^{j}$ are the analysis filters, $P^{j}$ and $Q^{j}$ are the synthesis filters. Valette's scheme [10] attempt to inverse the connectivity simplification to 1:4 subdivision as much as possible. This is for semi-regular regions in the irregular input meshes. If 4:1 simplification is not possible, it will be merged in groups of three or two faces, or leave some faces unchanged. Edge flips are performed when needed.

Fig. 1 shows the general concept of our approach. It is inspired from Kanai et 
al. [7] and Uccheddu [8]. In contrast with the previous approaches where the watermarking method was based on regular wavelet multiresolution analysis which was limited to meshes with 4:1 subdivision connectivity, we use the irregular wavelet analysis scheme [10], which supports 4:1, 3:1, 2:1, 1:1 merging. In addition, starting from the Lazy wavelet filter-bank (Eq. (1) - (3)) following [9] we build new filters $A^{j}, Q^{j}$ by the Lifting scheme in order to make the wavelet functions more orthogonal to the scaling functions in the 1-ring. By using irregular wavelet analysis scheme, our method can embed the watermark for both regular and irregular 3-D triangular meshes.

\subsection{Preprocessing for DWT of 3D surface meshes}

Unlike discrete wavelet transform (DWT) of 2D images, the wavelet coefficients of 3D meshes depend on the seed-triangle group merged during connectivity graph simplification. This artifact results from both irregular sampling and unusual scanning of the 3D surface. In addition, although the lossless reconstruction of integer coordinates meshes is possible and the reconstructed mesh from $M^{0}$ to $M^{J}$ is exact in terms of geometry, the original vertex indices were lost when processing with the irregular wavelet analysis [10].

Such a problem can be overcome by reordering the original model before irregular wavelet analysis. Our walking algorithm for reordering an irregular mesh is inspired both from Touma-Gotsman connectivity coder [11] and from the well-known backtracking algorithm for the visit of tree nodes. The reordering starts from a seed triangle which is determined from geometry criteria such as the triangle with the largest area, the farthest triangle from the model centroid. Connectivity information, for example, the triangle which has the greatest number of neighboring triangles within its $n$-ring, also could be good to use. After having processed the reordered mesh by watermark embedder, the resulting mesh should be reordered on the basis of the previously defined seed triangle group in order to recover the original vertex indices (Fig. 1). Reordering brings an additional computational cost, however, it can be an another interest for watermarking, because the reordering criterion can be also used as a secret key to protect the watermark over unauthorized erasing or multiple watermarking of wavelet coefficients.

It is very important to select the criterion which is insensitive against various attacks. In this paper, we use a connectivity criterion: the sum of the number of neighboring vertices within their 4-ring. All the manifold meshes can be processed by our walking algorithm. Fig. 2 describes our walking algorithm for reordering an irregular mesh. From the seed-face $f 1$, we define both focus vertex $v 1$ and walking vertex $v 3$ with the same criterion for the seed-face $f 1$. In addition, the first focus vertex should not have a boundary edge. All the visited faces and edges indices need to be stored in index buffers to process the exception handling, for example, a visited face, a boundary edge. Then, we walk to the neighbor face $f 2$ which shares $v 1$ and $v 2$ in $f 1$ and store $f 2$ and the edge $(v 1, v 2)$ (see Fig. 2(a)). The next vertex $v 4$ will be the next walking vertex. $f 3$ can be found with the same rule with the focus vertex $v 1$ and the new walking 


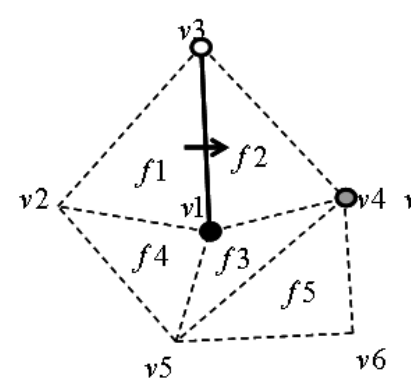

(a)

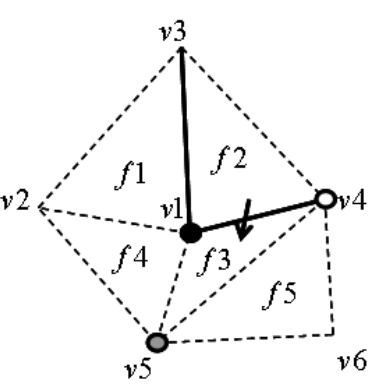

(b)

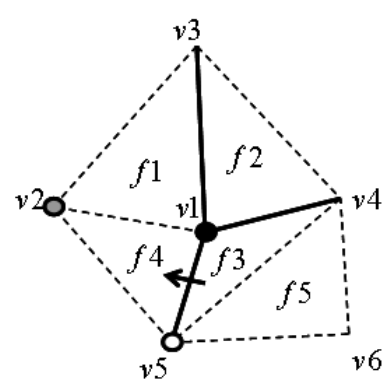

(c)

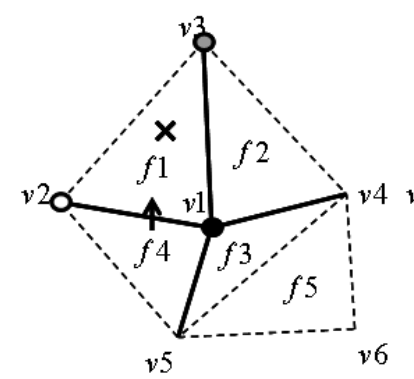

(d)

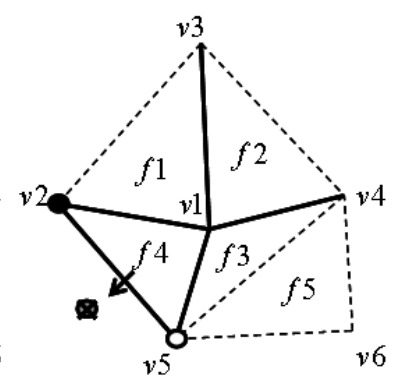

(e)

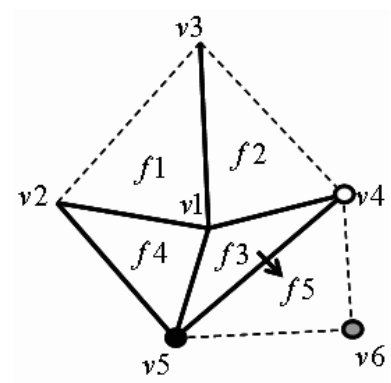

(f)

Fig. 2. Our walking algorithm for reordering where dark point is focus vertex, white point is walking vertex and gray point is the next vertex. The arrow means the direction to walk. Solid line depicts visited edges and dashed line is unvisited edges.

vertex $v 4$ (Fig. 2(b), Fig. 2(c)). If the neighbor face has been visited as shown in Fig. 2(d), we need to backtrace the last ordered face $f 4$ in the index buffer. For backtracing the buffer, we also need to switch the focus and the walking vertices. Boundary edges also can be treated by the same way as visited faces.

\subsection{Embedding Method}

Following Eq. (2), $D$ is the vector of wavelet coefficients.

$$
D^{j-1}=\left[d_{0}, d_{1}, \ldots, d_{N-1}\right]^{t}
$$

where, $d_{n}=\left[\begin{array}{lll}x_{n} & y_{n} & z_{n}\end{array}\right]^{t}$ and $N$ is the number of wavelet coefficients. We omit the resolution level $j-1$ for the sake of notational simplicity.

After we compute forward DWT of the mesh, we obtain the wavelet coefficient vector $D$. We convert the Cartesians coordinates of the coefficient $d_{n}$ to spherical coordinates $\left[\left\|d_{n}\right\|, \theta_{n}, \phi_{n}\right]^{t}$ where, $\left\|d_{n}\right\|$ denotes L2-norm of wavelet coefficients. Note that $\left\|d_{l}\right\|$ is invariant to rotation and translation attacks.

We split the $\left\|d_{n}\right\|$ into $I$ regular sections, called bins (Fig. 3). We index each $b i n^{i}$ from $i=0$ to $I-1$. We consider that the $\left\|d_{n}\right\|$ are uniformly distributed 


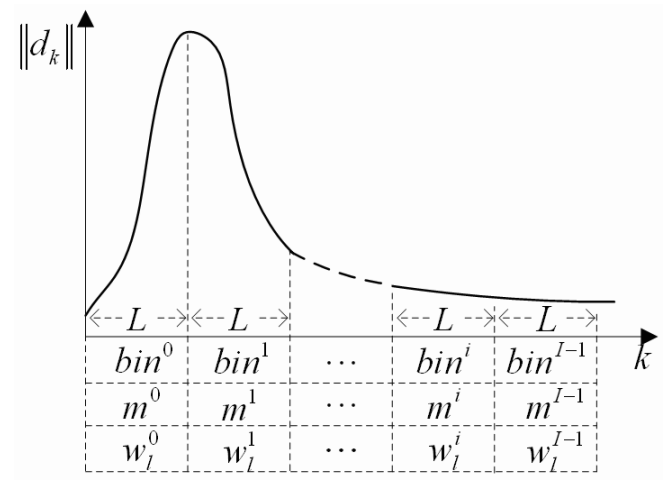

Fig. 3. Generation of bins.

and there are $L=N / I$ vectors in each bin. After that we generate one message bits per bin and a zero mean pseudo random sequence $p_{l}$ of length $L$ for all bins as follows,

$$
\begin{aligned}
& m^{i} \in\{-1,1\}, 0 \leq i<I \\
& p_{l} \in\{-1,1\}, 0 \leq l<L
\end{aligned}
$$

The watermark $w_{l}^{i}$ is generated from Eq. (5) and Eq. (6).

$$
w_{l}^{i}=m^{i} p_{l}, \quad 0 \leq i<I, 0 \leq l<L
$$

We embed $w_{l}^{i}$ into $\left\|d_{l}\right\| \in b_{i n}{ }^{i}$ in proportion to $\left\|d_{l}\right\|$ and the global strength factor $\beta$. $\beta$ can help to extract the watermark easily, but it has to be selected carefully because it also controls the visual quality after embedding the watermark.

$$
\left\|\tilde{d}_{l}^{i}\right\|=\left\|d_{l}\right\|+\beta\left\|d_{l}\right\| w_{l}^{i}
$$

Then, spherical coordinates $\left[\left\|\tilde{d}_{l}^{i}\right\|, \theta_{l} \phi_{l}\right]^{t}$ are changed into Cartesian coordinates $\left[\tilde{x}_{l}, \tilde{y}_{l}, \tilde{z}_{l}\right]^{t}=\tilde{d}_{l}^{i}$. Finally, we can get the watermarked mesh $\tilde{M}^{J}$ after inverse DWT (Eq. (3)) with $\tilde{d}_{l}^{i}$. Note that the inverse reordering of $\tilde{M}^{J}$ is required to recover the original indices of both vertices and faces as we mentioned in Section 2.2.

\subsection{Extraction Method}

We use a blind extraction scheme based on cross-correlation of the norm of wavelets coefficients of the attacked mesh $\tilde{\tilde{M}}^{J}$ with the pseudo random sequence. For the sake of notational simplicity we do not change the notation of wavelet coefficients. In other words the following can be considered as a mesh free of attack. According to Eq. (8) the cross correlation results in,

$$
\sum_{l \in b i n^{i}}\left\|\tilde{d}_{l}^{i}\right\| p_{l}=\sum_{l \in b i n^{i}}\left\|d_{l}\right\| p_{l}+\beta \sum_{l \in b i n^{i}}\left\|d_{l}\right\| w_{l}^{i} p_{l}
$$


From Eq. (5) and Eq. (6), $w_{l}^{i} p_{l}=m^{i} p_{l}^{2}=m^{i}$. In addition, due to both the zero mean pseudo random sequence and the uniform distribution in a bin, the first term of Eq. (9) cancels. Then, we can recover $m^{i}$ by computing the sign of Eq. (9).

$$
\operatorname{sign}\left[\sum_{l \in b i n^{i}}\left\|\tilde{d}_{l}^{i}\right\| p_{l}\right]=m^{i}
$$

The components of wavelet coefficients follow a generalized Gaussian pdf [10]. Then the pdf of the wavelet coefficient norms is close to a $\chi_{3}^{2}$ distribution [12]. It means that the distribution in the bins is not uniform. However, our approach remains effective in practice when the watermarked wavelet coefficient norms were limited to the minimum number of wavelet coefficients in the overall bins $L_{\text {min }}$. It can be enhanced by removing the first bin which is clearly out of the uniform hypothesis.

\section{$3 \quad$ Experimental Results}

In this section, we demonstrate the experimental results to show the effectiveness of our proposal. Since wavelet based approach is specific of the synchronization of the order of vertices and faces, we do not consider the topological attacks such as re-triangulation, simplification or remeshing [8]. We use four meshes in Fig. 3. Three of them are irregular meshes: Stanford Bunny (34834 vertices, 69451 faces), Davidhead (24085 vertices, 47753 faces), Hand (10196 vertices, 20261 faces), and the other is an semi-regular mesh, Head (6737 vertices, 13408 faces).

We first embedded one bit into Head to present the efficiency of transparency and robustness according to the watermark strength factor $\beta$ in both Fig. 5 and Table 1. Root mean square $(R M S)$ distance between the original mesh and the watermarked in $\%$ of the bounding box diagonal [13], estimates

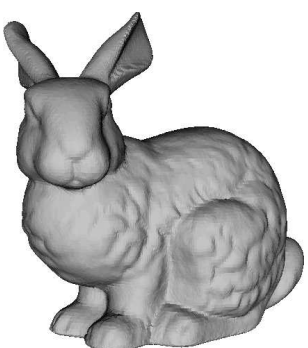

(a) Bunny

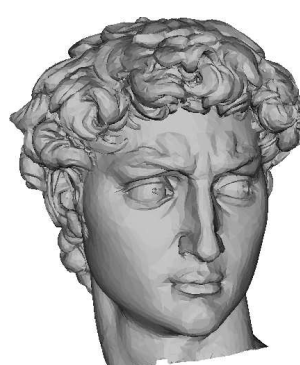

(b) Davidhead

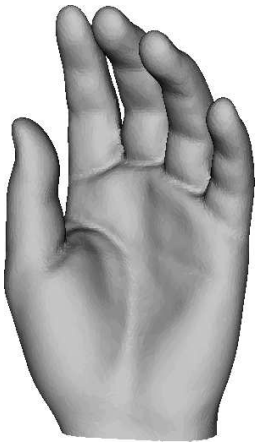

(c) Hand

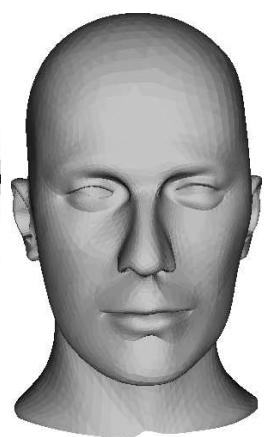

(d) Head

Fig. 4. Test models 


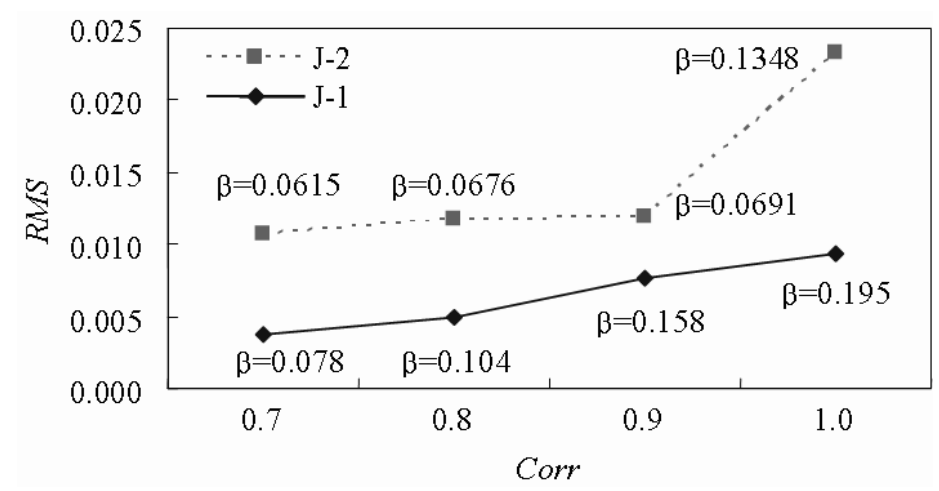

Fig. 5. Efficiency of transparency and robustness versus the strength factor $\beta$.

the visual quality. We use linear correlation (corr) between extracted message bits $\left(\tilde{m}^{i}\right)$ and designated message bits $\left(m^{i}\right)$ to measure the robustness. In the

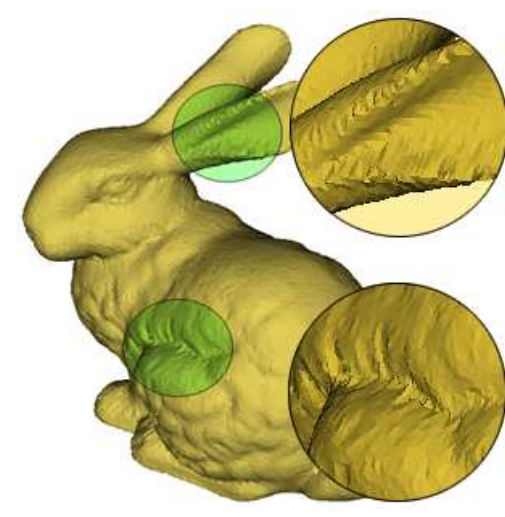

(a) $R M S$ distance $=0.015296$

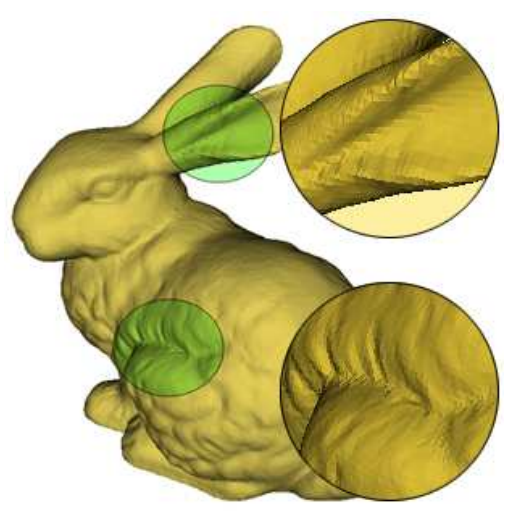

(b) $R M S$ distance $=0.015602$

Fig. 6. $R M S$ distance for Bunny according to embedding level (a) $J-1$ where $\beta=0.8$, and (b) $J-4$ where $\beta=0.1$.

Table 1. corr and $R M S$ distances without an attack.

\begin{tabular}{cccccccccc}
\hline \multirow{2}{*}{$\begin{array}{c}\text { Embedding } \\
\text { Level }\end{array}$} & \multirow{2}{*}{$\beta, I$} & \multicolumn{2}{c}{ Bunny } & Davidhead & Hand & \multicolumn{2}{c}{ Head } \\
\cline { 3 - 9 } & & corr & RMS & corr & RMS & corr & RMS & corr & $R M S$ \\
\hline$J-1$ & $0.3,125$ & 1.000 & 0.005 & 1.000 & 0.018 & 1.000 & 0.007 & 1.000 & 0.014 \\
\hline$J-1$ & $0.4,125$ & 1.000 & 0.007 & 1.000 & 0.024 & 1.000 & 0.009 & 1.000 & 0.019 \\
\hline$J-1$ & $0.4,265$ & 1.000 & 0.007 & 1.000 & 0.024 & 1.000 & 0.009 & 1.000 & 0.019 \\
\hline$J-2$ & $0.2,50$ & 1.000 & 0.009 & 1.000 & 0.020 & 1.000 & 0.010 & 1.000 & 0.035 \\
\hline Multilevel $^{\mathrm{a}}$ & - & 1.000 & 0.011 & 1.000 & 0.032 & 1.000 & 0.014 & 1.000 & 0.040 \\
\hline
\end{tabular}

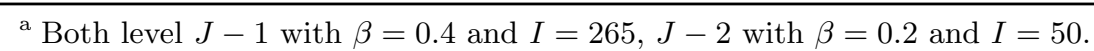


same level, robustness (corr) increases according to $\beta$, but $R M S$ distance also increases with $\beta$. Fig. 5 also shows that the wavelet coefficients of lower resolution level $J-2$ (dashed line) is more affected than higher resolution level $J-1$ (solid line).

To compare the transparency between two resolution levels, we embedded the watermark with different strength factor at each levels. In Fig. 6, although two models have similar $R M S$ distances, the watermark in the higher resolution level is more visible than in the lower resolution level, because the modification in the lower frequency component is less affected to high curvature area. It proves that the methods based on frequency domain are more effective than spatial domain methods when the application needs a good visual quality of the model after the watermarking.

$$
\operatorname{corr}\left(\tilde{m}^{i}, m^{i}\right)=\frac{\sum_{i=0}^{I-1}\left(\tilde{m}^{i}-\overline{\tilde{m}}^{i}\right)\left(m^{i}-\bar{m}^{i}\right)}{\sqrt{\sum_{i=0}^{I-1}\left(\tilde{m}^{i}-\tilde{\tilde{m}}^{i}\right)^{2}} \sqrt{\sum_{i=0}^{I-1}\left(m^{i}-\bar{m}^{i}\right)^{2}}}
$$

The capacity $I$ is dependent on the number of wavelet coefficients $N$ in our approach. It also can be varied by $\beta$. For the fair analysis of robustness, we embed the same number of bits with the same strength factor to all models. We give the result from single level at $J-1$ in Table 2 . For noise attack, we add a noise vector to each vertex. The amplitudes of the noise vectors are $0.2 \%$ and $0.45 \%$. We applied laplacian smoothing with the relaxation factor of 0.03 and the iteration 20 and 40 times each. corr increases according to $\beta$. But, if we increase $\beta$, it makes the watermark more visible. A small number of embedded bits gives better robustness.

Table 3 presents the result of multilevel embedding. We embedded 315 bits,

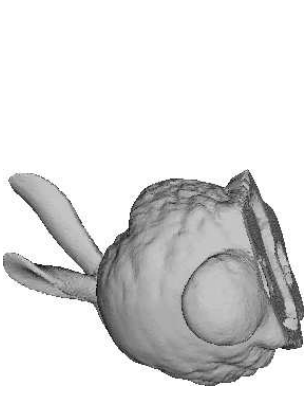

(a)

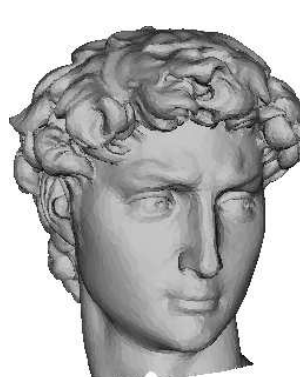

(b)

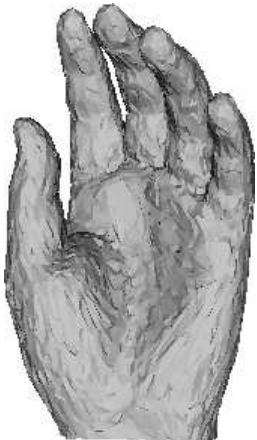

(c)

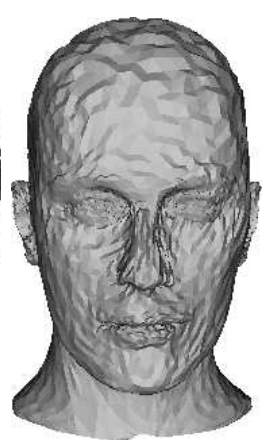

(d)

Fig. 7. Attacked models: (a) Affine transform, (b) Laplacian smoothing $0.03 \times 40$, (c) Progressive compression 8 bitplanes, (d) Additive noise $0.45 \%$. 
Table 2. Robustness against additive noise and laplacian smoothing attacks of single level at $J-1$

\begin{tabular}{lccccc}
\hline \multirow{2}{*}{$(\beta, I)$} & \multicolumn{4}{c}{ corr } \\
\cline { 3 - 6 } & & Bunny Davidhead Hand Head \\
\hline \multirow{2}{*}{ Noise $^{0.2 \%}$} & $0.3,125$ & 0.952 & 1.000 & 0.984 & 0.890 \\
& $0.4,125$ & 1.000 & 1.000 & 1.000 & 0.968 \\
& $0.4,265$ & 0.940 & 1.000 & 1.000 & 0.903 \\
\hline \multirow{2}{*}{ oise $^{0.45 \%}$} & $0.3,125$ & 0.744 & 1.000 & 1.000 & 0.615 \\
& $0.4,125$ & 0.856 & 1.000 & 1.000 & 0.744 \\
& $0.4,265$ & 0.751 & 1.000 & 0.992 & $0.601^{\mathrm{a}}$ \\
\hline \multirow{2}{*}{ Smooth $^{20}$} & $0.3,125$ & 0.920 & 1.000 & 0.906 & 0.936 \\
& $0.4,125$ & 1.000 & 1.000 & 1.000 & 1.000 \\
& $0.4,265$ & 0.947 & 1.000 & 0.970 & 0.962 \\
\hline \multirow{2}{*}{ Smooth $^{40}$} & $0.3,125$ & 0.795 & 0.968 & 0.744 & 0.588 \\
& $0.4,125$ & 0.888 & 1.000 & 0.808 & 0.728 \\
& $0.4,265$ & 0.706 & 0.917 & 0.753 & 0.751 \\
\hline
\end{tabular}

${ }^{\mathrm{a}} 0.518$ in Yin et al. [5].

(265 bits at level $J-1$ and 50 bits at level $J-2$ ), into all test models. The strength factors are $\beta=0.4$ at $J-1$ and $\beta=0.2$ at $J-2$, respectively. The table gives corr at each level and the average of both levels. For compression attack, we applied progressive geometry compression proposed by Valette [14]. It encodes the wavelet coefficients using a zerotree coder with a bitplane approach and reconstructs the mesh geometry progressively while the connectivity is kept unchanged. The numbers of bitplanes for compression were 8 and 9 , and a small number of bitplanes gives lower quality of the model. $J-2$ results a better robustness comparing with $J-1$ cause $D^{J-2}$ is less affected than $D^{J-1}$ on the other attacks. The table shows that the given strength factors are not sufficient to explain the robustness against all the attacks, but the capacity is still effective comparing with the previous work in Yin et al. [5] which uses non-blind detection.

We also tested similarity transform (ST) and affine transform and random connectivity reordering of both faces and vertex indices. Since L2-norm of wavelet coefficients is invariant to rotation and translation, our method that embeds into wavelet coefficient norms gives error-free detection on rotation and translation for both single level and multilevel. In addition, it is also effective on scaling attacks including non-uniform scaling. Random reordering attack can be recovered by the reordering before the DWT.

\section{Conclusion}

In this paper, we proposed a multiresolution analysis based watermarking method for 3D irregular meshes. The proposed embeds the watermark into L2-norm of wavelet coefficients in various multiresolution levels. Through the experiment, we proved that a vertex and face re-ordering process as pre-processing in both 
Table 3. Robustness against additive noise $\left(A N^{\text {amplitude }}\right)$ and laplacian smoothing $\left(L S^{\text {numbers of iteration }}\right)$ and progressive compression $\left(P C^{\text {numbers of bitplane }}\right)$, random connectivity reordering $(R R)$, similarity transform $(S T)$, uniform scaling $\left(U S^{(x, y, z)}\right)$, affine transform $(R S T)$ of multilevel embedding.

\begin{tabular}{|c|c|c|c|c|c|c|c|c|c|}
\hline & \multirow{2}{*}{ Level } & \multicolumn{2}{|c|}{ Bunny } & \multicolumn{2}{|c|}{ DavidHead } & \multicolumn{2}{|c|}{ Hand } & \multicolumn{2}{|c|}{ Head } \\
\hline & & corr & Avg. & corr & $A v g$. & corr & Avg. & corr & Avg. \\
\hline \multirow{2}{*}{$A N^{0.2 \%}$} & $J-1$ & 0.940 & \multirow{2}{*}{0.970} & 1.000 & \multirow{2}{*}{1.000} & 1.000 & 1000 & 0.903 & \multirow{2}{*}{0.951} \\
\hline & $J-2$ & 1.000 & & 1.000 & & $\overline{1.000}$ & 1.000 & 1.000 & \\
\hline \multirow{2}{*}{$A N^{0.45 \%}$} & $J-1$ & 0.751 & \multirow{2}{*}{0.876} & 1.000 & \multirow{2}{*}{1.000} & 1.000 & 1000 & 0.601 & \multirow{2}{*}{0.801} \\
\hline & $J-2$ & 1.000 & & $\overline{1.000}$ & & $\overline{1.000}$ & 1.000 & $\overline{1.000}$ & \\
\hline \multirow{2}{*}{$L S^{20}$} & $J-1$ & 0.955 & \multirow{2}{*}{0.977} & 1.000 & \multirow{2}{*}{1.000} & 0.962 & $000 ?$ & 0.962 & \multirow{2}{*}{0.981} \\
\hline & $J-2$ & 1.000 & & 1.000 & & $\overline{0.842}$ & 0.902 & 1.000 & \\
\hline \multirow{2}{*}{$L S^{40}$} & $J-1$ & 0.698 & \multirow{2}{*}{0.809} & 0.917 & \multirow{2}{*}{0.900} & 0.723 & 0666 & 0.766 & \multirow{2}{*}{0.863} \\
\hline & $J-2$ & $\overline{0.919}$ & & $\overline{0.883}$ & & $\overline{0.610}$ & 0.000 & $\overline{0.960}$ & \\
\hline \multirow{2}{*}{$P C^{8}$} & $J-1$ & 0.992 & \multirow{2}{*}{0.996} & 1.000 & \multirow{2}{*}{1.000} & 0.992 & 0006 & 0.584 & \multirow{2}{*}{0.792} \\
\hline & $J-2$ & 1.000 & & 1.000 & & $\overline{1.000}$ & 0.990 & $\overline{1.000}$ & \\
\hline \multirow{2}{*}{$P C^{9}$} & $J-1$ & 0.947 & \multirow{2}{*}{0.974} & 1.000 & \multirow{2}{*}{1.000} & 1.000 & 1000 & 0.940 & \multirow{2}{*}{0.970} \\
\hline & $J-2$ & 1.000 & & $\overline{1.000}$ & & 1.000 & 1.000 & $\overline{1.000}$ & \\
\hline \multirow{2}{*}{$R R$} & $J-1$ & 1.000 & 1000 & 1.000 & 1000 & 1.000 & 1000 & 1.000 & ( \\
\hline & $J-2$ & 1.000 & 1.000 & 1.000 & 1.000 & $\overline{1.000}$ & 1.000 & $\overline{1.000}$ & 1.000 \\
\hline$S T$ & $J-1$ & 1.000 & 1000 & 1.000 & 1000 & 1.000 & 1000 & 1.000 & 1000 \\
\hline SI & $J-2$ & 1.000 & 1.000 & 1.000 & 1.000 & 1.000 & 1.000 & $\overline{1.000}$ & 1.000 \\
\hline$U S^{(2.0,1.0,1.0)}$ & $J-1$ & 1.000 & 1000 & 1.000 & 1000 & 1.000 & 1000 & 1.000 & 1000 \\
\hline & $J-2$ & 1.000 & & 1.000 & 1.000 & 1.000 & 1.000 & 1.000 & 1.000 \\
\hline & $J-1$ & 1.000 & 1000 & 1.000 & 1000 & 1.000 & 1000 & 1.000 & 1000 \\
\hline & $J-2$ & 1.000 & 1.000 & 1.000 & 1.000 & 1.000 & 1.000 & 1.000 & 1.000 \\
\hline & $J-1$ & 0.992 & 0.006 & 0.992 & 0006 & 1.000 & 0,080 & 1.000 & 0080 \\
\hline & $J-2$ & 1.000 & 0.990 & $\overline{1.000}$ & 0.990 & $\overline{0.960}$ & 0.980 & 0.960 & 0.980 \\
\hline $\mathrm{BST}^{\mathrm{d}}$ & $J-1$ & 1.000 & 1000 & 1.000 & 1000 & 1.000 & & 1.000 & 1000 \\
\hline In & $J-2$ & 1.000 & 1.000 & $\overline{1.000}$ & 1.000 & $\overline{1.000}$ & 1.000 & 1.000 & 1.000 \\
\hline $\mathrm{BST}^{\mathrm{e}}$ & $J-1$ & 0.992 & 0996 & 0.992 & 0.006 & 1.000 & 108 & 1.000 & 1000 \\
\hline $10 \mathrm{~N}$ & $J-2$ & 1.000 & 0.550 & 1.000 & 0.550 & 1.000 & 1.000 & 1.000 & 1.000 \\
\hline
\end{tabular}

${ }^{\mathrm{a}} 0.518$ in Yin et al. [5],

${ }^{\mathrm{b}} 0.786$ in Yin et al. [5].

c Similarity Transform from user's random input. 0.779 in Yin et al. [5].

${ }^{\mathrm{d}} R^{\left(30^{\circ}, 60^{\circ}, 90^{\circ}\right)}, S^{(2.0,1.1,1.7)}, T^{(5,10,15)}$

е $R^{\left(90^{\circ}, 30^{\circ}, 60^{\circ}\right)}, S^{(1.1,1.7,2.0)}, T^{(-5,10,-15)}$

watermark embedding and extraction make our proposal be robust against connectivity reordering attacks. We also showed that multiresolution embedding has more capacity while keeping the robustness against connectivity reordering as well as various kinds of geometrical attacks such as lossy compression and affine transform. 


\section{References}

1. Cox, I., Kilian, J., Leighton, T., Shamoon, T.: Secure spread spectrum watermarking for multimedia. IEEE Trans. Image Processing 6 (1997) 1673-1687

2. Fridrich, J., Goljan, M., Du, R.: Lossless data embedding-new paradigm in digital watermarking. EURASIP J. Appl. Signal. Process. 2002 (2002) 185-196

3. Praun, E., Hoppe, H., Finkelstein, A.: Robust mesh watermarking. In: Proceedings of the 26th annual conference on Computer graphics and interactive techniques. (1999) 49-56

4. Ohbuchi, R., Masuda, H., Aono, M.: Watermarking three-dimensional polygonal models. In: Proceedings of the fifth ACM international conference on Multimedia, ACM Press (1997) 261-272

5. Yin, K., Pan, Z., Shi, J., Zhang, D.: Robust mesh watermarking based on multiresolution processing. Computers and Graphics 25 (2001) 409-420

6. Cho, J.W., Kim, M.S., Prost, R., Chung, H.Y., Jung, H.Y.: Robust watermarking on polygonal meshes using distribution of vertex norms. In Cox, I.J., Kalker, T., Lee, H.K., eds.: IWDW. Volume 3304 of Lecture Notes in Computer Science., Springer (2004) 283-293

7. Kanai, S., Date, D., Kishinami, T.: Digital watermarking for 3d polygon using multiresolution wavelet decomposition. In: Proc. Sixth IFIP WG 5.2 GEO-6, Tokyo, Japan (1998) 296-307

8. Uccheddu, F., Corsini, M., Barni, M.: Wavelet-based blind watermarking of 3d models. In: MM\&Sec '04: Proceedings of the 2004 multimedia and security workshop on Multimedia and security, ACM Press (2004) 143-154

9. Lounsbery, M., DeRose, T.D., Warren, J.: Multiresolution analysis for surfaces of arbitrary topological type. ACM Transactions on Graphics 16 (1997) 34-73

10. Valette, S., Prost, R.: Multiresolution analysis of irregular surface meshes. IEEE Trans. Visual. Comput. Graphics 10 (2004) 113-122

11. Touma, C., Gotsman, C.: Triangle mesh compression. In: Graphics Interface. (1998) 26-34

12. Payan, F., , Antonini, M.: Multiresolution 3d mesh compression. In: IEEE Int. Conf. on Image Processing ICIP'02. Volume 2. (2002) 245-248

13. Aspert, N., Santa-Cruz, D., Ebrahimi, T.: Mesh: Measuring errors between surfaces using the hausdorff distance. In: Proceedings of the IEEE International Conference on Multimedia and Expo. Volume I. (2002) $705-708$ http://mesh.epfl.ch.

14. Valette, S., Gouaillard, A., Prost, R.: Compression of 3d triangular meshes with progressive precision. Computer \& Graphics, Special Issue on Compression 28 (2004) 35-42 